\title{
Impact of Two Touch Types on Preterm's Behavioral Reaction
}

\author{
MARIANA E. SHEHATA, D.N.Sc.*; SHADIA R. EL-GUINDY, D.N.Sc.*; HANAN M. RASHAD, D.N.Sc.*; \\ FATEN H. ABD EL-AZEIM, Ph.D.** and HEBA H. ABOUHUSSEIN, M.D.*** \\ The Department of Pediatric Nursing, Faculty of Nursing* and The Department of Pediatric Growth \& Development, \\ Faculty of Physical Therapy** and The Department of Pediatric Medicine, Faculty of Medicine***, Cairo University, Egypt
}

\begin{abstract}
Background: Touch is one of the first strong positive senses that develop in neonates.

Aim of Study: The aim of the current study was to evaluate the impact of two touch types on Preterm's behavioral reaction.

Subjects and Methods: Quasi-experimental research design was utilized for the current study.

Setting: The current study was conducted at Maternal and Newborn Hospital in Kaser Al-Aini Hospital, Cairo University.

Sample: A convenient sample was consisted of ninety preterm neonates divided into three equal groups "thirty for each", control, gentle human touch and Yakson. Each preterm neonate received both interventions provided for twice a day for consecutive five days. Each session lasting for fifteen minutes. The control group received routine nursing care, while the other two groups received either gentle human touch or Yakson before routine care.

Results: The current study revealed that a statistically significantly difference of both intervention groups regarding to Anderson score compared to that of control group. The intervention groups had improved the sleep state for preterm neonates than that of control.

Conclusion: Gentle human touch and Yakson interventions improved behavioral state of preterm neonates.

Recommendation: The current study recommended that both types of touch safe and an effective and in routine care for preterm neonates.
\end{abstract}

Key Words: Two types - Touch - Preterm's - Behavioral reaction.

\section{Introduction}

A PREMATURE neonate is deprived of in-utero sensory experiences which are essential for normal brain development and rather is exposed to unusual sensory stimuli in the NICU that pose risk to the

Correspondence to: Dr. Mariana E. Shehata, The Department of Pediatric Nursing, Faculty of Nursing, Cairo University, Egypt developing brain in terms of adverse neurodevelopmental outcomes [1]. Organ systems are insufficiently developed to fully support extra-uterine life resulting in increased biological risk for complications of prematurity. In the normal human fetus, several organ systems mature between 34 and 37 weeks, and the fetus reaches adequate maturity by the end of this period [2]

Touch has the unique effects on the human organism. All neonates including the smallest and sickest need loving human contact. Stressors can have negative effect on the neonate's immune systems, where the tactile stimulation can be favorable since it reduces tension and improves immune system. Tactile stimulation or massage therapy began as a sacred system of natural healing. Tactile stimulation is part of massage therapy. While doing tactile stimulation the skin is being stimulated. The skin is the largest organ of the body. It has countless nerve endings for touch; pain and pressure that are accountable for various tactile sensations that take part a significant responsibility in the maturity of the neonate. The skin has intimate contact with the Central Nervous System (CNS) [3]

Gentle human touch and Yakson massage therapy could improve sleep status in premature infants, and in comparison, to gentle human touch, Yakson massage is more effective in the in the stress relief of premature infants [4]. Furthermore, [5] evaluated the effects of Yakson therapy, gentle touch and routine nursing care on premature infant by measuring different variables, such as urinary cortisol and norepinephrine hormone concentrations.

Touch or massage therapy as non-invasive procedure has positive effect on physical and developmental outcomes of premature neonates including weight gain that is associated with shorter 
hospital stays, promotion of neurologic and neuromotor development, decreased stress behavior, improved infant parent attachment, improved sleep, reduced rates of nosocomial infection and mortality rate of hospitalized premature neonates. Consequently, massage has been proposed as a way of facilitating development and growth of neonates through its effects on increasing their blood flow, heart rate, digestion, and immunity [6,7]

\section{Aim of study:}

The aim of the current study was to evaluate the impact of two touch types on Preterm's behavioral reaction.

\section{Significance of the study:}

This study will provide an evidence based to nursing and other health care professionals for optimizing the preterm neonates sensory experience, decreasing stress, improving physiological and behavioral state outcomes. As well as providing guidance and recommendations that should be reflected on pediatric nursing education. More over providing evidence-based data that can develop nursing practice and research in the field of neonatal nursing to improve quality of neonatal nursing care.

Developmentally supportive care is endorsed by researches from several scientific fields, including neuroscience, developmental and family psychology, medicine and nursing. In recent years, research examining the effectiveness of individualized, developmentally supportive care to newborn infants in the NICU has demonstrated a variety of positive effects. It significantly improved medical outcomes, including reduced dependence on respiratory support, improved weight gain, earlier accomplishment of nipple feedings, shortened lengths of stay, and reduced costs of care [8]

\section{Research hypothesis:}

HO- There will be no significant differences between the two relaxation methods of touch "gentle human touch and Yakson" on behavioral state of Preterms'.

H1- Preterm who will receive either gentle human or Yakson touch will exhibit lower behavioral state scores (ABSS) than control group.

\section{Research design:}

A quasi-experimental research design (pre-post study) was utilized for the current study. This type of research design involves one or more groups of subjects observed before and after the implementation of an intervention [9]

\section{Material and Methods}

The study was conducted in Neonatal Intensive Care Unit (NICU) at Maternal and Newborn Hospital in Kaser Al-Aini Hospital, Cairo University. The NICU $2014-2015$. Present at the fourth floor of Maternal and Newborn Hospital that consists of four rooms the first one, consists of admission room for well-born babies for observation, its capacity is about six incubators and four servo, the second one, include two partitions each one consists of six incubators, the third one, for isolation for severe infected cases (four incubators) and the last room for grower till discharge.

Sample:

A convenient sample of 90 neonates were included and divided equally into three groups. 30 for control group that left to hospital routine care, 30 for (GHT.) group and 30 for Yakson group.

\section{Inclusion criteria:}

- Gestational age between 32-<37 weeks at birth.

- Both genders.

- Apgar score more than 6 at 1 and 5 minutes.

- Age: After 7 days of birth for physiological stabilization [10].

\section{Exclusion criteria:}

- Preterm neonate with respiratory problems and connected with mechanical ventilator.

- Preterm neonate with diseases such as asphyxia, sepsis, neurological problems and other congenital anomalies such as central nervous system.

- Preterm neonate undergoing surgery.

Tools:

To achieve the aim of the study the following tools were used for data collection:

1-Preterm neonate data sheet include: Birth data of the neonate that recorded from the neonate medical file e.g date of birth, gestational weeks, etc.

2- Anderson Behavioral State Scale (ABSS): Standardized tool that was developed by Anderson [11]. This scale determines the behavioral state of neonate based on observations of respiratory regularity, opening or closing of the eyes, limb and trunk activity, and the intensity of crying. This scale measures 12 neonate's behavioral states including 1 ; regular quiet sleep, 2 ; irregular quiet sleep, 3; active sleep, 4; very active sleep, 5; drowsy, 6; alert inactivity,7; quiet awake, 8; 
active awake, 9; very active awake, 10; fussing, 11 ; crying and 12; hard crying. Scores from 1 to 5 indicate that the neonate is sleeping. Scores 6-8 indicate that the neonate is awake and calm and in the most suitable state for nursing activity. Scores from 9 to 12 indicate that the infant is in a state of restless activity or fussiness, which take substantial energy.

3- Gentle human touch protocol.

4- Yakson protocol.

\section{Procedure:}

After gaining the primary approval of Research Ethical Committee from the Faculty of Nursing, Cairo University, an official permission obtained from the director and head of Department of Maternal and Newborn Hospital in Kaser Al-Aini Hospital, Cairo University to carry the current study, a written informed consent was obtained from the parents of selected neonates. Participation was voluntary, and parents were allowed to withdraw the preterm neonates from the study at any time without any effect on care given to their neonates.

In addition, the researcher carried out the intervention and measured physiological parameters and behavioral responses in the study and control groups. Yakson massage and GHT were performed by the researcher twice a day for 15 minutes in the (morning 9-11 Am) and (evening 3-5Pm) for 5 consecutive days.

Control group: The researcher assessed and recorded the physiological parameters and Anderson score-for ethical consideration firstly collectedtwo minutes before initiation of routine nursing care and two minutes after finishing care twice a day for five consecutive days the same as intervention group.

Gentle Human Touch (GHT): Researcher placed the fingertips of one hand above the eyebrow line with the palm touching the preterm neonates crown while the other hand will be rested on the lower abdomen of the neonate encompassing the waist and the hip for 15 minutes.

Yakson touch: Session lasted for 15 minutes hand resting 5 minutes, gentle caressing 5 minutes, and hand resting again 5 minutes. During providing of Yakson, the palms and all fingers of the researcher constantly maintained close contact with the preterm neonate up to the limit that they did not feel pressure.
Data collected started from the beginning of June 2014 till the end of May 2015.

\section{Pilot study:}

A pilot study was done on nine preterm infants to test the feasibility of the study and was included in the study.

\section{Validity and reliability: \\ A tool of ABSS is valid and its reliability was 0.96 .}

\section{Ethical consideration:}

A primary and final ethical approval was obtained from the Research Ethical Committee in the Faculty of Nursing, Cairo University. All premature neonates' parents were informed about the purpose of the study to obtain their acceptance to share in the study, the research investigator explained aim of the study, benefits of it, and duration of the study. Written consent obtained from neonate's mothers or fathers and they informed that participation in the current study was voluntary, the data collected was used only for research purpose, anonymity and confidentiality of each participant assured and the parent's right to withdrawal their neonate from the study at any time without given reasons.

\section{Data analysis:}

Data entry and statistical analysis were done using SPSS 20.0 statistical software package. Data were presented using descriptive statistics in the form of frequencies and percentages for qualitative variables, and means and standard deviations for quantitative variables. Quantitative continuous data were compared using paired $t$-test for dependent (pre-post) groups. The non-parametric MannWhitney and Kruskal-Wallis tests were used for comparing independent three means and more than two means, respectively. Spearman rank correlation was used for assessment of the inter-relationships among quantitative variables and ranked ones. In order to identify the independent predictors of changes in Vineland scores, multiple linear regression analysis was used, and analysis of variance for the full regression models was done. Statistical significance was considered at $p$-value $<0.05$.

\section{Results}

Table (1) represented that all preterm neonates in three groups had statistically similar baseline characteristics. No statistically significant differences were detected in the mean of the three groups regarding their gestational age, postnatal age and Agar score after one and five minutes $p=(0.09$, 
$0.23,0.35 \& 0.46$ respectively). Majority of preterm neonates were delivered via cesarean section $80 \%$ in GHT, $70 \%$ in Yakson and $73.3 \%$ in control group. Nearly all of cases in three groups were feeders and the majority of them on formula feeding $(96.7 \%$ \& $96.6 \%$ in control, $96.7 \%$ \& $93.1 \%$ in GHT and $100 \%$ \& $86.7 \%$ in Yakson group). No statistically significant difference were detected in the three groups regarding labor, on feeding and milk type ( $p=0.66,0.60 \& 0.17$ respectively).

Fig. (1) showed that no statistically significant difference in the three groups regarding their gender $p=0.19$.

Fig. (2) showed that the majority of preterm neonate diagnosis was RDS in all three groups in GHT, Yakson and control $(93.30 \%, 100 \%$ and $93.30 \%$ respectively).
Table (2) illustrated that before intervention, no statistically significant difference were detected in the average mean scores of three groups in control, GHT, Yakson $(6.2 \pm 2.3,6.1 \pm 2.1,6.2 \pm 1.0$, respectively, $p=0.88$ ) while after intervention it was decreased significantly in both GHT and Yakson than control $(3.0 \pm 1.2,3.0 \pm 1.1,7.2 \pm 1.3$, respectively $p=<0.001 * * *)$. Results showed that average mean of Anderson sleep state after five days of intervention for both GHT and Yakson preterm neonate sleeps comfort $(100 \%)$ than those of control group (13.3\%).

Table (3) represented that before intervention, no statistically significant difference was detected in mean Anderson score but after intervention, a highly significant difference was found among the three groups between 1 st $\& 5$ th day $p=(0.40 \&$ 0.001 respectively).

Table (1): Preterm neonates characteristics in the three study groups in percentage distribution $(\mathrm{n}=90)$.

\begin{tabular}{|c|c|c|c|c|c|c|c|}
\hline \multirow{3}{*}{$\begin{array}{l}\text { Preterm neonate } \\
\text { characteristics }\end{array}$} & \multicolumn{6}{|c|}{ Groups } & \multirow{3}{*}{$\begin{array}{c}p- \\
\text { value }\end{array}$} \\
\hline & \multicolumn{2}{|c|}{ Control n=30 } & \multicolumn{2}{|c|}{ GHT n=30 } & \multicolumn{2}{|c|}{ Yaskon $n=30$} & \\
\hline & No. & $\%$ & No. & $\%$ & No. & $\%$ & \\
\hline \multicolumn{8}{|c|}{ Gestational weeks: } \\
\hline $32-33$ & 21 & 70.0 & 12 & 40.0 & 19 & 63.3 & \\
\hline $34-35$ & 7 & 23.3 & 13 & 43.3 & 7 & 23.3 & \\
\hline $36-<37$ & 2 & 6.7 & 5 & 16.7 & 4 & 13.3 & \\
\hline Mean $\pm \mathrm{SD}$ & \multicolumn{2}{|c|}{$33.0 \pm 1.3$} & \multicolumn{2}{|c|}{$33.8 \pm 1.5$} & \multicolumn{2}{|c|}{$33.4 \pm 1.3$} & 0.09 \\
\hline \multicolumn{8}{|c|}{ Post-natal age/day: } \\
\hline $7-14$ & 6 & 20.0 & 4 & 13.3 & 3 & 10.0 & \\
\hline $15-21$ & 10 & 33.3 & 15 & 50.0 & 18 & 60.0 & \\
\hline $22-28$ & 14 & 46.7 & 11 & 36.7 & 9 & 30.0 & \\
\hline Mean $\pm \mathrm{SD}$ & \multicolumn{2}{|c|}{$23.6 \pm 9.2$} & \multicolumn{2}{|c|}{$19.7 \pm 6.4$} & \multicolumn{2}{|c|}{$19.7 \pm 6.9$} & 0.23 \\
\hline \multicolumn{8}{|c|}{ Apgar score (1 min): } \\
\hline$<7$ & 25 & 83.3 & 25 & 83.3 & 26 & 86.7 & \\
\hline $7+$ & 5 & 16.7 & 5 & 16.7 & 4 & 13.3 & \\
\hline Mean \pm SD & \multicolumn{2}{|c|}{$4.4 \pm 1.7$} & \multicolumn{2}{|c|}{$4.4 \pm 2.0$} & \multicolumn{2}{|c|}{$4.0 \pm 1.9$} & 0.35 \\
\hline \multicolumn{8}{|c|}{ Apgar score (5min): } \\
\hline$<7$ & 1 & 3.3 & 2 & 6.7 & 0 & 0.0 & \\
\hline $7+$ & 29 & 96.7 & 28 & 93.3 & 30 & 100.0 & \\
\hline Mean $\pm S D$ & \multicolumn{2}{|c|}{$7.9 \pm 1.0$} & \multicolumn{2}{|c|}{$7.7 \pm 1.0$} & \multicolumn{2}{|c|}{$7.9 \pm 1.0$} & 0.46 \\
\hline \multicolumn{8}{|l|}{ Delivery mode: } \\
\hline Cesarean & 22 & 73.3 & 24 & 80.0 & 21 & 70.0 & \\
\hline Vaginal & 8 & 26.7 & 6 & 20.0 & 9 & 30.0 & 0.66 \\
\hline \multicolumn{8}{|l|}{ On feeding: } \\
\hline Yes & 29 & 96.7 & 29 & 96.73 & 30 & 100.0 & \\
\hline No & 1 & 3.3 & 1 & 3.3 & 0 & 0.0 & 0.60 \\
\hline \multicolumn{8}{|l|}{ Milk type: } \\
\hline Formula & 28 & 96.6 & 27 & 93.1 & 26 & 86.7 & \\
\hline Breast & 1 & 3.3 & 1 & 3.3 & 3 & 10.0 & 0.17 \\
\hline Mixed & 1 & 3.4 & 2 & 6.9 & 1 & 3.3 & \\
\hline
\end{tabular}

GHT: Gentle Human Touch. 
Table (2): Comparison of Anderson mean scores in the three study groups before and after intervention days.

\begin{tabular}{|c|c|c|c|c|c|c|c|c|c|c|c|c|c|c|}
\hline \multirow{4}{*}{$\begin{array}{l}\text { Anderson scores } \\
\text { before and after } \\
\text { intervention days }\end{array}$} & \multicolumn{6}{|c|}{ Before intervention } & \multicolumn{7}{|c|}{ After intervention } & \multirow{4}{*}{$\begin{array}{c}p- \\
\text { value }\end{array}$} \\
\hline & \multicolumn{6}{|c|}{ Groups } & \multirow{3}{*}{$\begin{array}{c}p- \\
\text { value }\end{array}$} & \multicolumn{6}{|c|}{ Groups } & \\
\hline & \multicolumn{2}{|c|}{$\begin{array}{c}\text { Control } \\
(\mathrm{n}=30)\end{array}$} & \multicolumn{2}{|c|}{$\begin{array}{c}\text { GHT } \\
(n=30)\end{array}$} & \multicolumn{2}{|c|}{$\begin{array}{l}\text { Yaskon } \\
(\mathrm{n}=30)\end{array}$} & & \multicolumn{2}{|c|}{$\begin{array}{l}\text { Control } \\
(\mathrm{n}=30)\end{array}$} & \multicolumn{2}{|c|}{$\begin{array}{c}\text { GHT } \\
(n=30)\end{array}$} & \multicolumn{2}{|c|}{$\begin{array}{l}\text { Yaskon } \\
(\mathrm{n}=30)\end{array}$} & \\
\hline & No. & $\%$ & No. & $\%$ & No. & $\%$ & & No. & $\%$ & No. & $\%$ & No. & $\%$ & \\
\hline Day 1: & & & & & & & & & & & & & & \\
\hline (Sleep) 1-5 & 15 & 50.0 & 18 & 60.0 & 17 & 56.7 & & 9 & 30.0 & 26 & 86.7 & 24 & 80.0 & \\
\hline (Awake) 6-8 & 10 & 33.3 & 6 & 20.0 & 5 & 16.7 & 0.88 & 15 & 50.0 & 3 & 10.0 & 6 & 20.0 & $<0.001$ \\
\hline (Restless) 9-12 & 5 & 16.7 & 6 & 20.0 & 8 & 26.7 & & 6 & 20.0 & 1 & 3.3 & 0 & 0.0 & $* * *$ \\
\hline Mean \pm SD & \multicolumn{2}{|c|}{$6.2 \pm 2.3$} & \multicolumn{2}{|c|}{$6.1 \pm 2.1$} & \multicolumn{2}{|c|}{$6.5 \pm 2.6$} & & \multicolumn{2}{|c|}{$6.9 \pm 2.1$} & \multicolumn{2}{|c|}{$3.3 \pm 2.2$} & \multicolumn{2}{|c|}{$3.2 \pm 2.1$} & \\
\hline \multicolumn{15}{|l|}{ Day 2: } \\
\hline (Sleep) 1-5 & 11 & 36.7 & 8 & 26.7 & 14 & 46.7 & & 5 & 16.7 & 27 & 90.0 & 26 & 86.7 & $<0.001$ \\
\hline (Awake) 6-8 & 15 & 50.0 & 13 & 43.3 & 11 & 36.7 & & 19 & 63.3 & 3 & 10.0 & 3 & 10.0 & $* * *$ \\
\hline (Restless) 9-12 & 4 & 13.3 & 9 & 30.0 & 5 & 16.7 & 0.44 & 6 & 20.0 & 0 & 0.0 & 1 & 3.3 & \\
\hline Mean \pm SD & \multicolumn{2}{|c|}{$6.4 \pm 2.3$} & \multicolumn{2}{|c|}{$7.0 \pm 2.2$} & \multicolumn{2}{|c|}{$6.4 \pm 2.2$} & & \multicolumn{2}{|c|}{$7.3 \pm 2.2$} & \multicolumn{2}{|c|}{$3.2 \pm 1.6$} & $3.4 \pm$ & & \\
\hline Day 3: & & & & & & & & & & & & & & \\
\hline (Sleep) 1-5 & 13 & 43.3 & 12 & 40.0 & 7 & 23.3 & & 8 & 26.7 & 28 & 93.3 & 27 & 90.0 & \\
\hline (Awake) 6-8 & 10 & 33.3 & 16 & 53.3 & 21 & 70.0 & & 11 & 36.7 & 2 & 6.7 & 3 & 10.0 & $<0.001$ \\
\hline (Restless) 9-12 & 7 & 23.3 & 2 & 6.7 & 2 & 6.7 & 0.84 & 11 & 36.7 & 0 & 0.0 & 0 & 0.0 & \\
\hline Mean \pm SD & $6.4 \pm$ & & $6.3 \pm$ & & $6.4 \pm$ & & & $7.3 \pm$ & & $3.4 \pm$ & & $3.3 \pm$ & & \\
\hline Day 4: & & & & & & & & & & & & & & \\
\hline (Sleep) 1-5 & 12 & 40.0 & 18 & 26.7 & 17 & 56.7 & & 9 & 30.0 & 29 & 96.7 & 30 & 100.0 & \\
\hline (Awake) 6-8 & 16 & 53.3 & 20 & 66.7 & 12 & 40.0 & & 14 & 46.7 & 1 & 3.3 & 0 & 0.0 & \\
\hline (Restless) 9-12 & 2 & 6.7 & 2 & 6.7 & 1 & 3.3 & 0.11 & 7 & 23.3 & 0 & 0.0 & 0 & 0.0 & $<0.001$ \\
\hline Mean \pm SD & $5.9 \pm$ & & $6.4 \pm$ & & $5.7 \pm$ & & & $7.0 \pm$ & & $3.0 \pm$ & & $2.7 \pm$ & & $* * *$ \\
\hline Day 5: & & & & & & & & & & & & & & \\
\hline (Sleep) 1-5 & 13 & 43.3 & 14 & 46.7 & 17 & 56.7 & & 5 & 13.3 & 30 & 100.0 & 30 & 100.0 & \\
\hline (Awake) 6-8 & 11 & 36.7 & 14 & 46.7 & 12 & 40.0 & 0.29 & 15 & 50.0 & 0 & 0.0 & 0 & 0.0 & \\
\hline (Restless) 9-12 & 6 & 20.0 & 2 & 6.7 & 1 & 3.3 & & 11 & 36.7 & 0 & 0.0 & 0 & 0.0 & $<0.001$ \\
\hline Mean \pm SD & $6.4 \pm$ & & $5.9 \pm$ & & $5.5 \pm$ & & & $7.8 \pm$ & & $2.2 \pm$ & & $2.1 \pm$ & & $* * *$ \\
\hline Average (5 days): & & & & & & & & & & & & & & \\
\hline (Sleep) 1-5 & 9 & 30.0 & 6 & 20.0 & 6 & 20.0 & & 4 & 13.3 & 30 & 100.0 & 30 & 100.0 & \\
\hline (Awake) 6-8 & 19 & 63.3 & 23 & 76.7 & 23 & 76.7 & 0.88 & 21 & 70.0 & 0 & 0.0 & 0 & 0.0 & \\
\hline (Restless) 9-12 & 2 & 6.7 & 1 & 3.3 & 1 & 3.3 & & 5 & 16.7 & 0 & 0.0 & 0 & 0.0 & $<0.001$ \\
\hline Mean \pm SD & $6.2 \pm$ & & $6.1 \pm$ & & $6.2 \pm$ & & & $7.2 \pm$ & & $3.0 \pm$ & & $3.0 \pm$ & & $* * *$ \\
\hline
\end{tabular}

Table (3): Comparisons of the changes in Anderson scores for the three study groups between the 1 st and 5 th day before and after the intervention.

\begin{tabular}{|c|c|c|c|c|}
\hline \multirow{2}{*}{$\begin{array}{l}\text { Physiological parameters and } \\
\text { Anderson scores } \\
\text { Changes between } 1 \text { st \& } 5 \text { th day }\end{array}$} & \multicolumn{3}{|c|}{ Groups } & \multirow[b]{2}{*}{$p$} \\
\hline & $\begin{array}{c}\text { Control } \\
\text { Mean } \pm \text { SD }\end{array}$ & $\begin{array}{c}\text { GHT } \\
\text { Mean } \pm S D\end{array}$ & $\begin{array}{c}\text { Yaskon } \\
\text { Mean } \pm \text { SD }\end{array}$ & \\
\hline Before Anderson scores & $0.1 \pm 2.8$ & $-0.2 \pm 3.0$ & $-0.9 \pm 3.1$ & 0.40 \\
\hline After Anderson scores & $0.8 \pm 2.7$ & $-1.0 \pm 2.3$ & $-1.2 \pm 2.3$ & $0.001 * * *$ \\
\hline $\begin{array}{ll}\text { GHT } & : \text { Gentle Human Touch. } \\
* & : \text { Statistically significant at } p \\
* * * & : \text { Statistically significant at } p\end{array}$ & & & & \\
\hline
\end{tabular}




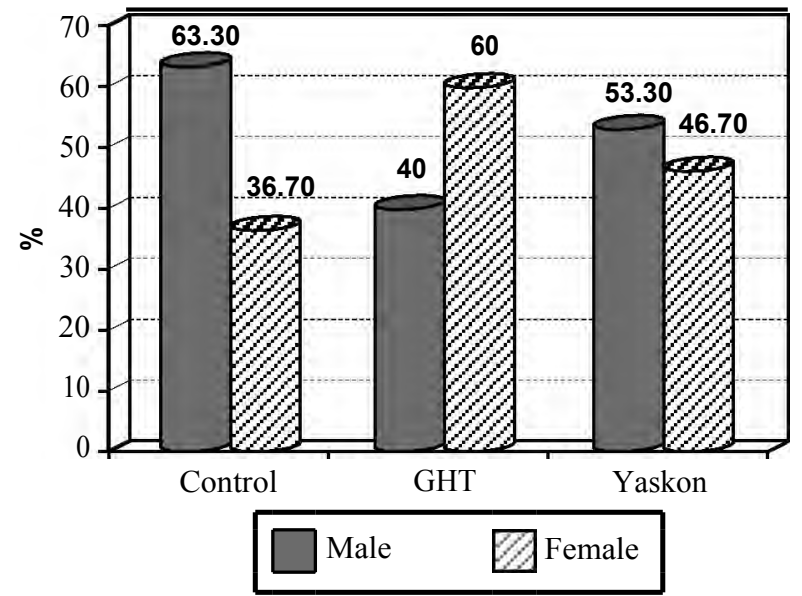

Fig. (1): Preterm neonate gender in the three groups in percentage distribution $(\mathrm{n}=90)$.

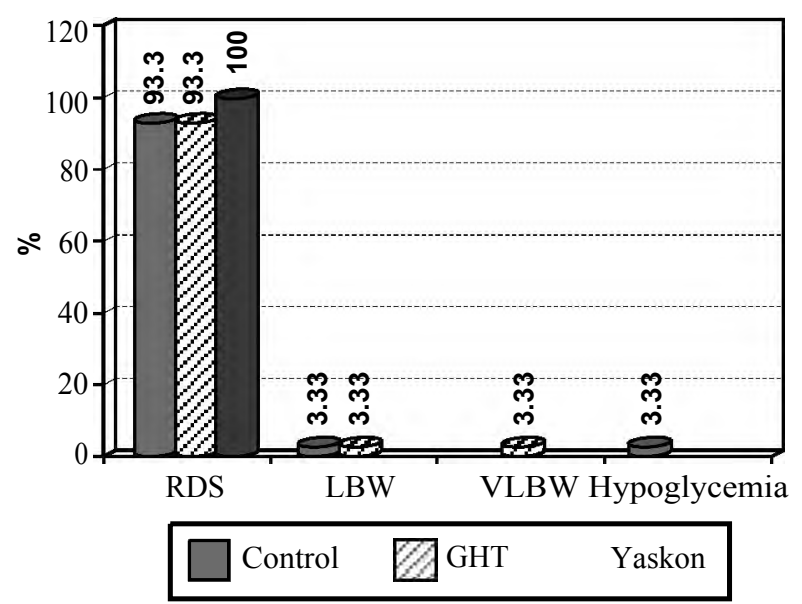

Fig. (2): Preterm neonate diagnosis in percentage distribution $(n=90)$.

RDS : Respiratory Distress Syndrome.

LBW : Low Birth Weight.

VLBW : Very Low Birth Weight.

GHT : Gentle Human Touch.

\section{Discussion}

Regarding gestational age the current study findings revealed that no significance differences were found in the means of the three groups when the majority of them their gestational age ranged from thirty two to thirty five. The current study findings goes in the same line with [12] who concluded in his review of "Birth weight versus gestational age as a predictor of viability of extremely low birth weight babies" that more than two thirds of premature neonates were between thirty-two to thirty five weeks. This study was full agreement with [13] who studied the "effect of oil massage on anthropometric parameters and behavioral state of stable low birth weight neonates", that carried out in Kaser El-Ani Hosoital, Cairo University and reported that more than two thirds of low birth neonates were premature, and their gestational age ranged between thirty-two to thirty-five weeks.

Concerning to gender of preterm neonates, the current study represented that more than half of control and Yakson groups were boys and more than half of the preterm neonates were females in the GHT group with no differences in the three groups. The findings were in accordance with [14] in Mansoura University Children's Hospital who studied "the effectiveness of developmentally supportive positioning on preterm infants' pain response at Neonatal Intensive Care Units" and reported that more than half of preterms in studied and control groups were males. In contrast the current study finding disagree with [15], who studied "effect of prefeeding oral stimulation program on feeding performance of preterm infants in the NICUs of Cairo University Hospitals" and reported that more than half of preterms were females in both groups. Also, [16] who studied "A MetaAnalysis of Preterm Infant Massage: An Ancient Practice with Contemporary Applications" reported that more than half were females and less than half were males.

In Egypt, preterm neonates suffering from RDS admission rate is considered a high percentage in relation to all other diagnosis according to [17] The result of the current study revealed that the majority of neonates were diagnosed with RDS in the three groups and this finding in accordance with [18], who studied "leading causes of neonatal mortality in neonatal Intensive Care Unit in Kaser Al-Ani Hospital", and [19], and they reported that all neonates were preterms and more than half of them were diagnosed as RDS.

In the current study, the majority of preterms were formula fed, which is consistent with study of [20] that done in Denmark who stated that majority of preterm neonates admitted to NICUs was on bottle feeding, the cause was associated with multiple factors e.g. infants, mothers and clinical practices at the NICUs, maternal factors strongly associated with inadequate breastfeeding duration and mothers with low education. Factors in clinical practice as rooming-in of the mother and the infant for a few days before discharge in order to establish breastfeeding both day and night was common at all NICUs. Breast milk expression was an important issue at NICUs. From researcher's point of view the cause might be no rooming in, unavailability of mother in clinical setting, inexperienced mothers about importance of breast milk and fear of dealing with their preterm neonates. 
According to the results of the current study, there was a significant difference between the ABSS scores of premature neonates receiving GHT, Yakson massage therapy and control neonates after the intervention ( $p=0.001)$. Furthermore, scores of sleep status in infants of the GHT and Yakson interventions group were higher compared to the neonates in the control group. Previous studies in this regard have confirmed the efficacy of GHT and Yakson massage interventions on the sleep status of premature infants [21].

After five days of GHT and Yakson interventions in the current study, scores of waking and restlessness decreased in premature infants, which is consistent with the findings of previous studies confirming the positive effects of GHT and Yakson massage therapy on the status of waking and restlessness in premature infants of study [22] who discussed the "effects of Yakson Therapeutic Touch on the Behavioral Response of Premature Infants". This finding indicated that use of GHT and Yakson massage could increase the comfort of premature infants, reduce their stress and help with relaxation during admission at the NICU.

Finally, this study evidenced that GHT and Yakson massage had decreases active sleep, increase quiet sleep and decreased behavioral on premature neonate.

\section{Conclusion:}

According to the results of the present study, it was concluded that GHT and Yakson touch had significant effects on the behavioral response of premature neonates, resulting in increased scores of sleep status, as well as decreased restlessness state. Findings of the current study rejected the null hypothesis and supported the hypothesis one.

\section{Recommendations:}

Based on the findings of the current study, the following recommendations were suggested:

- Add Anderson Score tool in the nursing record.

- Yakson and GHT could be one of the safe interventions that nurses use for preterm neonates in order to enhance their development.

- Collaboration and continuing education of the staff in the NICU (doctors, nurses, etc.) are vital to improve the quality of care provided for preterm neonates.

\section{References}

1- RAMACHANDRAN S. and DUTTA S.: Early Developmental Care Interventions of Preterm Very Low Birth Weight Infants. Indian Pediatrics, 50 (15): 765-70, 2013.
2- BENZIES K.M., MAGILL-EVANS J.E., HAYDEN K.A. and BALLANTYNE M.: Key components of early intervention programs for preterm infants and their parents: A systematic review and meta-analysis, BMC Pregnancy and Childbirth, 13 (1): S10 http://www.biomedcentral.com/ 1471-2393/13/S 1/S 10, 2013.

3- LIVINGSTON L.M.M.: Effectiveness of Tactile and Auditory Stimulation on Physiological Parameters among Preterm Neonates. Doctorate published thesis Available at: repository-tnmgrmu.ac.in/2712/1/ 14040552014majella livingston.pdf, 2014.

4- VICKERS A., OHLSSON A., LACY J.B. and HORSLEY A.: Massage for promoting growth and development of preterm and/or low birth-weight infants. Cochrane Database Syst. Rev., (2): CD000390, 2004.

5- IM H., KIM E. and CAIN C.K.: Acute effects of yakson and gentle human touch on the behavioral state of preterm infants. J. Child Health Care, 13 (3): 212-26, 2009.

6- AKHAVAN K.: Effect of body massage on increase of low birth weight neonates' growth parameters. Iran J. Reprod. Med., 11 (7): 583588, 779, 2013.

7- ANG J.Y., JORGE L.L., MATHUR A., THOMAS R., ASMAR B.I., SAVASAN S., et al.: A Randomized Placebo-Controlled Trial of Massage Therapy on the Immune System of Preterm Infants, Pediatrics, 130 (6), pp. 154958, 2012.

8- MOHAMMED S.A., BAYOUMI M.H. and MAHMOUD F.S.: The Effect of Developmentally Supportive Care Training Program on Nurses' Performance and Behavioral Responses of Newborn Infants, Journal of Education and Practice ISSN 2222-1735 (Paper) ISSN 2222-288X (Online) Vol. 5, No. 6, Available at: www.iiste.org , 2014.

9- POLIT D. and BECK C.: Essential of Nursing Research. Appraising Evidence for Nursing Practice, 7 th ed. London. Walters Klawer. P.P. 100-5, 2011.

10- MODRCIN-TALBOTT M.A., HARRISON L.L., GROER M.W. and YOUNGER M.S.: Moderate versus light pressure massage therapy leads to greater weight gain in the mothers. Journal of Perinatal and Neonatal Nursing, 16 (3): 69-83, 2003.

11- ANDERSON G.C.: Anderson behavioral state scoring system cited in published doctorate thesis in Cong, (2006) Kangaroo Care for Analgesia in Preterm Infants Undergoing Heel Stick Pain, Available at www. https:// . etd.ohiolink.edu/!etd.send file?accession=case1134405 075\&disposition-online, 1996.

12- MOSTAFA A.: Birth weight versus gestational age as a predictor of viability of extremely low birth weight babies, Saudi Journal of Obesity, 5 (1): Pp: 1-2. Available from http://www.saudijobesity.com/article.asp?issn=23472618: year $=2017$; volume $=5$; issue $=1$; spage $=1$; epage $=2$; aulast $=$ Abolfotouh, 2017.

13- MAHMOUD H.S.: Effect of Oil Massage Therapy on Anthropometric Parameters and Behavioral State of Stable Low Birth Weight Neonates, unpublished doctorate thesis submitted to Faculty of Nursing, Cairo University, P.P.: 48-50, 2016. 
14- ABUSAAD F.E., ABD EL-AZIZ R.A. and NASEF N.A.: The Effectiveness of Developmentally Supportive Positioning on Preterm Infants' Pain Response at Neonatal Intensive Care Units, American Journal of Nursing Science; 6 (1): 63-71. Available at: http://www. sciencepublishinggroup.com/j/ajns doi: 10.11648/j.ajns.20170601.18 at 1/5/2017, 2017.

15-ABD EL-HAMID H.: Effect of prefeeding oral stimulation program on feeding performance of preterm infants in the NICUs of Cairo University Hospitals, unpublished doctorate thesis, Faculty of Nursing, Cairo University, Pp. 76-80, 2015.

16- BADR L.K., ABDALLAH B. and KAHALE L.: A MetaAnalysis of Preterm Infant Massage: An Ancient Practice with Contemporary Applications, 40 (6): Wolters Kluwer Health, 2015.

17- Statistics Department of Kaser AL-Ani Hospital, Cairo University. Unpublished setting hospital statistic office, 2016.

18-EL-BEHAIRY S.A.: Leading causes of neonatal mortality in neonatal Intensive Care Unit in Kaser Al-Aini Hospital.
Unpublished thesis for partial fulfillment of master degree in pediatrics, Faculty of medicine, Cairo University, P.P. 96-7, 2013.

19-BAYOMI O.R. and EL-NAGGER N.S.: Effect of applying massage therapy on physical, Behavioral Development. Addison--_Wesley Avaiable at: http://dx.doi.org/10.5430/ jnep.v5n10p105 accesed on 17/4/2015, 2015.

20- MÅSTRUP R.: Breastfeeding of preterm infants. Associated factors in infants, mothers, and clinical practice. Department of Health Sciences, Lund University, 2014.

21- BIJARI B.B., IRANMANESH S., ESHGHI F. and BANESHI M.R.: Gentle Human Touch and Yakson: The Effect on Preterm's Behavioral Reactions. International Scholarly Research Network ISRN Nursing Volume, Article ID 750363, 6 pages doi: 10.5402/2012/750363, 2013.

22- ESHGHI F., IRANMANESH S., BIJARI B.B., BORHANI F. and JAHROMI M.M.: Effects of Yakson Therapeutic Touch on the Behavioral Response of Premature Infants, J. Babol. Univ. Med. Sci., Vol. 17, Issu 10, Oct., P: 1521, 2015.

\section{تآثير طريقتان للمس على رد الفعل السلوكى للمبتسرين}

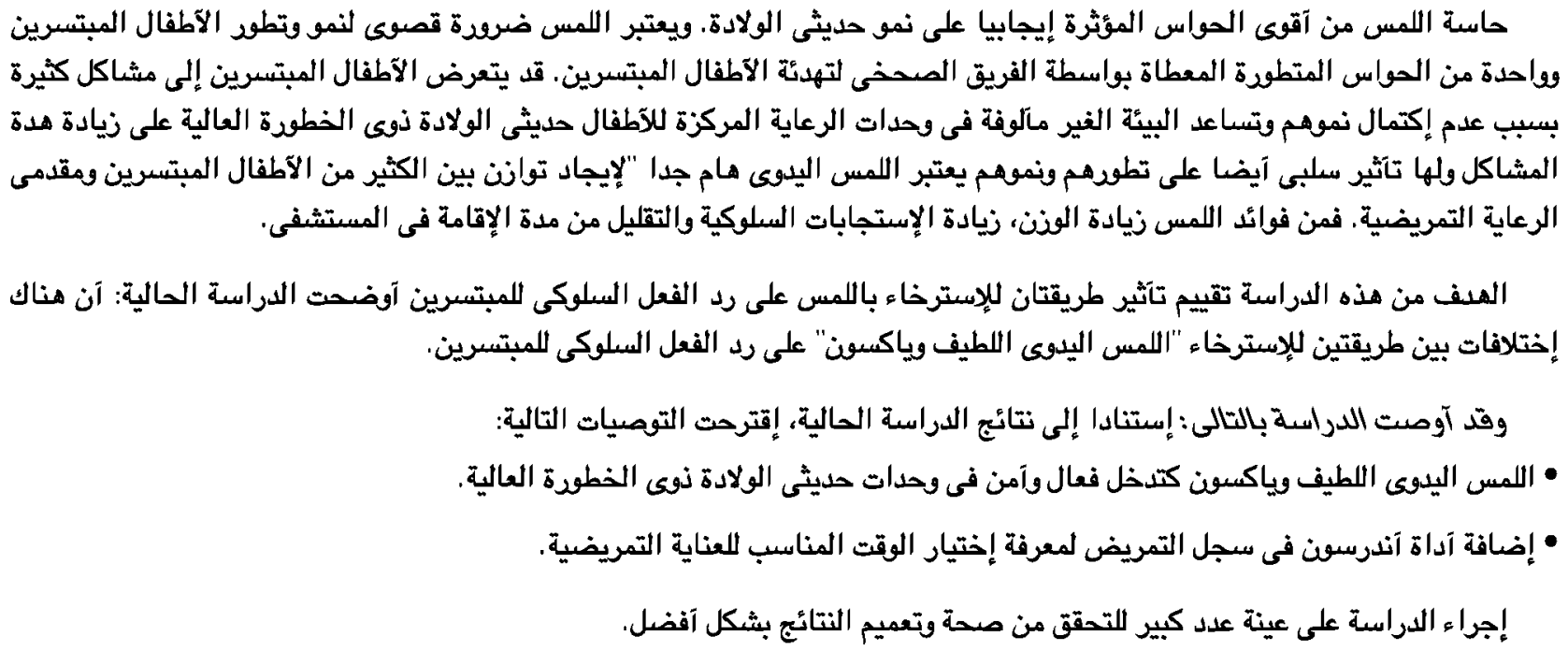

